\title{
VII. Elliptic and other interference with reflecting gratings
}

\section{Barus Ph.D. LL.D.}

To cite this article: C. Barus Ph.D. LL.D. (1911) VII. Elliptic and other interference with reflecting gratings , Philosophical Magazine Series 6, 22:127, 118-129, DOI: 10.1080/14786440708637103

To link to this article: http://dx.doi.org/10.1080/14786440708637103

Published online: 20 Apr 2009.

Submit your article to this journal $๘$

Џll Article views: 2

Q View related articles ¿ 
lowest key of the instrument a small weight sufficient to keep the damper off the string. Then set this string vibrating by plucking it with the fingers. The sound thus produced will last about 10 or 15 seconds, but the string will continue to vibrate for another 30 seconds or longer, and during the latter part of the time no sound will be audible.

The string vibrates in two planes: one parallel to the surface of the sound-board, and the other at right-angles to it. We have seen that the vibration of the string is transmitted to the sound-board most effectively by the pressing and pulling of the string on the bridge at right-angles to the surface of the sound-board. The vibration of the string in this plane is rapidly absorbed by the sound-board and therefore the audible sound rapidly ceases, but the vibration parallel to the surface of the sound-board does not set the latter in vibration to any appreciable extent, and therefore the vibration in this plane continues a much longer time and no sound is heard. By sliding a straight-edge along the face of the adjoining strings and gradually bringing it over the vibrating string, it will be found that the latter will hardly touch the straight-edge, thus showing the vibration to be in one plane.

14 City Road, London, E.C.

IIarch 23,1911 .

VII. Elliptic and other Interference with Reflecting Gratings. $B y$ C. Barus, Ph.D., LL.D., Professor of Physics at Brown University, Providence, U.S.A.*

1. FIRST METHOD.-There are two or three typical cases in the use of reflecting gratings for the production of interferences in the spectrum, each of which shows peculiarly interesting features. The first of these is given in fig. 1 , and corresponds closely to the method described for transmission gratings in a preceding paper $t$. If $\mathrm{L}$ is the source of light and $\bar{M}$ a glass plate grating, it was shown that plane mirrors in the positions $G_{m}$ and $G_{n}$, each reflecting a spectrum from $M$, produce elliptical interference whenever the rays returned after passing $M$ by transmission and reflexion, respectively, are made to overlap in the spectrum, under suitable conditions.

* Communicated by the Author. Abridged from a Report to the Carnegie Institution of Washington, D.C. Cf. Note in 'Science,' January 20, 1911.

+ Phil. Mag. July 1910. 
The present method is the converse of this, since the gratings and the opaque mirrors change places. Parallel rays from $L$ strike the plate of glass $M$ and the component

Fig. 1.

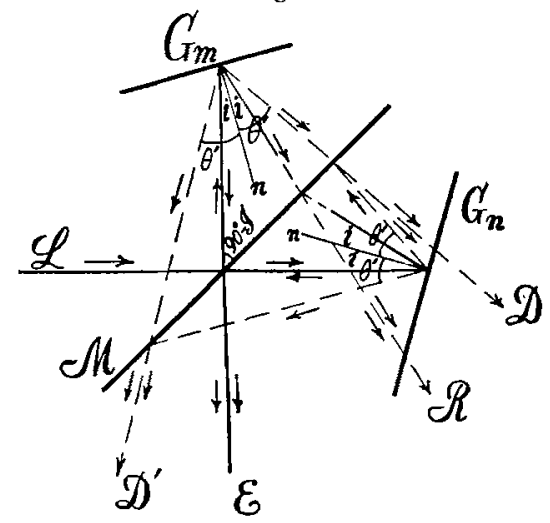

rays reach identical reflecting gratings $G_{m}$ and $G_{n}$, placed symmetrically with respect to $M$ at an angle $i$ to the $\mathrm{E}$ and $L$ directions. The undeviated rays pass off eccentrically at $\mathbf{R}$ and are not seen in the telescope at $\mathrm{E}$. They may, however, be seen in an auxiliary telescope pointed in the line R, and they then facilitate the adjustments. Rays diffracted at the angle $2 i$, however, are respectively transmitted and reflected by $\mathrm{M}$ and interfere in the telescope in the line E. Similarly rays diffracted at an angle $\theta^{\prime}>i$ interfere in the line D.

To make the adjustment it is sufficient to bring the Fraunhofer lines in the two spectra seen at $\mathrm{E}$ into complete coincidence, horizontally and vertically. Coincidence of slit images at $R$ (at least vertically) aids in the same result. It is also necessary that the rulings on $G_{m}$ and $G_{n}$ and the slit should be parallel, or that the images of slit and spectra shall lie between the same horizontals in the field. One of the gratings, $G_{n}$, may now be moved parallel to itself by the micrometer screw until the elliptic interferences appear. If the plate $\mathbf{M}$ is not half silvered there are three groups of these, as described in the preceding papor. Each group passes from the initial degree of extreme fineness, through inaximum size, to a final degree, for a play of the screw of about $1 \mathrm{~mm}$. There is the usual radial motion of the fringes, together with the drift through the spectrum as a whole. To bring out the set of solitary ellipses, the silvered surface of $M$ should be towards the light and remote from the eye. 
As a rule the adjustment is difficult, as an extra condition is imposed in the parallelism of the slit and the rulings of the gratings. The ellipses are liable to be coarse with their axes oblique, clearer in some parts of the spectrum than in others, unless means are provided for placing the rulings accurately parallel. Even when well adjusted they are rather polygonal than rounded in their contours. They are about as strong with non-silvered glass $M$ as with half-silvered glass ; but in view of the multiple spectra, the adjustment is much more difficult in the former case.

It has been suggested that the white slit images must appear eccentrically in the direction $R$. Hence, if a special telescope is directed in this line, the final adjustment is reached on coincidence of the proper slit images, provided the rulings of the gratings and the slit are parallel.

For $\theta^{\prime}>i$ the second series of interference spectra occurring at $D$ eccentrically are broader, but only on perfect adjustment do they occur simultaneously with the other set. In fact, since for the preceding case $i=\theta$, or

$$
2 \sin i=\lambda / \mathrm{D} \text {, }
$$

and in the presen case

therefore

$$
\sin \theta^{\prime}-\sin i=\lambda / \mathrm{D},
$$

$$
\sin \theta^{\prime}=3 \sin i=3 \sin \theta \text {. }
$$

There is also an available set in the second order to the left of $\mathrm{E}$. In the gratings used above, $D$ lies in front of $G_{n}$, being nearer the $\mathrm{E}$ than the $\mathrm{L}$ direction.

2. Inversion of the Method.-The occurrence of the undeviated ray $R$ suggests another method. For if the white ray $R$ is reversed, i. e. comes from an eccentric collimator, slit images will bo seen in telescopes at $L$ and $\mathbf{E}$, whereas overlapping spectra will appear in the direction $\mathrm{D}^{\prime}$ eccentrically and in the lines $R$ and $R^{\prime}$. One of the latter may be lost in the collimator. The former occurs for the same angle $\theta^{\prime}$, so that

$$
\sin \theta^{\prime}=3 \sin i .
$$

Moreover, if $\mathrm{I}=45^{\circ}$ is the angle of incidence of $\mathrm{L}$ upon $\mathrm{M}$ when sodium light is taken, so that

$$
\theta^{\prime}=26^{\circ} 14^{\prime}, \quad i=8^{\circ} 28^{\prime},
$$

the $\mathrm{R}, \mathrm{D}, \mathrm{D}^{\prime}$ rays make angles $2 i, \theta^{\prime}+i, \theta^{\prime}-i$, respectively, with the $\mathrm{E}$ direction; or the sum of the angles at $\mathrm{D}$ and $\mathrm{D}^{\prime}$ 
with the $\mathrm{E}$ line is $2 \theta^{\prime}$, their difference $2 i$, and the rays $\mathrm{D}$, $\mathrm{R}, \mathrm{D}^{\prime}$ intersect at a common centre on $\mathrm{G}_{m}$. Hence, if we place the plane of $G_{m}$ at the centre of the spectrometer and arrange $M$ and $G_{n}$ eccentrically, the angles may be measured as before.

3. Resolution of the Slit Image.-If the sharp white images of the slit in a Michelson apparatus for the case in which the incident light consists of parallel white rays from a collimator, be accurately superimposed, and the opaque mirrors be set at the proper distances from the semi-transparent mirror by the micrometer, the slit image may itself be viewed through a grating and will then show elliptic interferences in all the spectra. The apparatus is here eccentric, while the grating (either transmitting or reflecting) must be at the centre of the spectrometer, if angles are to be measured. The same is true for any of the other superimposed white slit images in the above or the earlier experiments, and may even be repeated with successive transmitting gratings. It is interesting to note that the position of the centre of ellipses is at the same wave-length in all the spectra, though the form of ellipses may differ enormously. The same phenomenon may thus be seen by a number of observers at the same time, each looking through his own telescope.

4. Third Method. Parallel Gratings.-In this case the two halves of the grating treated are displaced parallel to themselves, from their original co-planar position in the grating, from which they are cut. Their mounting is thus something like the case of the two black plates of Fresnel's mirror apparatus, one of the plates being adapted for displacement parallel to itself.

Fig. 2.

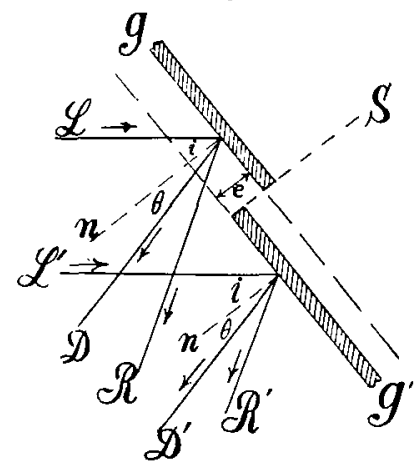

In fig. $2, g$ and $g^{\prime}$ show the two halves of the reflecting grating, cut along the plane $\mathrm{S}$, normal to the plates and 
parallel to the rulings. The half $g^{\prime}$ is provided with a micrometer screw, so that it may be successively moved from the position $g^{\prime}$ in fig. 2 to the position $g^{\prime}$ in fig. 3 , through all

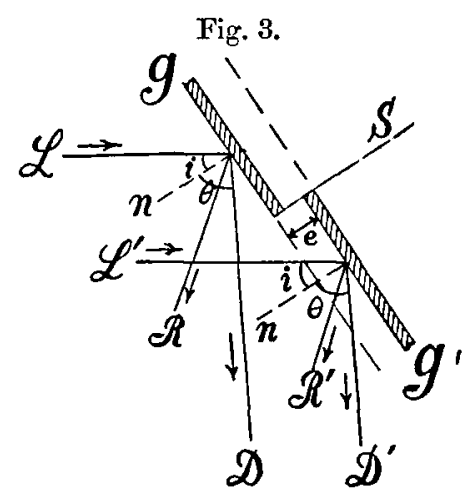

intermediate positions, while the half $g$ remains stationary. Each of the halves $g$ and $g^{\prime}$ is controlled by three adjustment screws (horizontal and vertical axes of rotation), to secure complete parallelism of the faces of the grating. Each, moreover, may be rotated around a horizontal axis to place the lines parallel to the slit of the collimator. The duplex. grating is mounted on a spectrometer as is usual for reflexion. Finally, each half may be raised and lowered and moved horizontally to and fro, parallel to itself, so that the half gratings when coplanar may approximately reproduce the original grating.

After each of the spectra is clear as to Fraunhofer lines, the interferences here in question are produced by bringing these lines (the D lines for instance) into perfect coincidence, horizontally and vertically. Under these circumstances if the distance apart, $e$, is suitably chosen, the interference fringes will appear throughout the spectrum. These consist of an approximately equidistant series of lines parallel to the slit, i. e. vertical lines, which are finer, ccet. par., as the breadth of the crack at $\mathrm{S}$ between the gratings is larger. They may be increased from the extreme fineness as they enter the range of visibility, to a maximum coarseness (in the above experiments) of about 3 to 5 minutes of arc per fringe, after which they vanish. They cannot in practice be passed through infinite size; neither can they be produced symmetrically on the two sides of the adjustment for infinite size. They cannot, in other words, be changed from the positive to tho negative condition of appearance. 
The occurrences are in fact as follows: if, as in fig. 2, $i>\theta$ (parallel white rays coming from $\mathrm{L}$ and $\mathrm{L}^{\prime}, \mathrm{R}$ and $\mathrm{R}^{\prime}$ being reflected, $\mathrm{D}$ and $\mathrm{D}^{\prime}$ diffracted rays for the normal $n$ ), the grating $g^{\prime}$ must be in advance or forward of $g$. If now the airspace $e$ is reduced micrometrically, $g^{\prime}$ retreating, the lines travel in a given direction (from left to right) through the spectrum, while at the same time they grow continually larger until for a minimum value of $e$ still positive, they vanish as a whole. The period of indistinctness before evanescence is not marked.

On the other hand, if $\theta^{\prime}>i$, as in fig. 3, the grating $g^{\prime}$ must be to the rear of $g$ and the airspace $e$ is throughout negative. If this is now decreased numerically the lines travel through the spectrum in the opposite direction to the preceding case, while at the same time they coarsen until they vanish as a whole, as before. The grating $g^{\prime}$ is still behind $g$ when this occurs.

Finally, if for any suitable value of $e$ the grating $g^{\prime}$ is moved in its own plane without rotation away from $g$, so as to widen the crack at $S$ between them, the fringes grow continually finer until they pass beyond visibility, and vice versa; i. e., as the crack at $\mathrm{S}$ is made smaller the lines continually coarsen.

5. Nature of the Evanessence.-The fact that the lines vanish as a whole and almost suddenly after reaching their maximum distance apart is very peculiar, as is also the fact that they cannot be passed through infinite size or appear symmetrically on both sides of this adjustment. To investigate this case I provided both the collimator and the telescope with slits so that the parts of the grating $g$ and $g^{\prime}$ from which the interfering pencils come might be investigated.

If a single vertical slit about $1 \mathrm{~mm}$. wide is passed from right to left towards the objective of the telescope, a black line passes across the field of the spectrum, which line is merely the image of the crack at $S$. Let the green rays, for instance, come from the edge of both gratings $g$ and $g^{\prime}$, whereas the red rays and the violet rays come from but a single grating. Now when the space $e$ is diminished, the black band in the green gradually vanishes, and in its place appear the coarsest fringes producible. When the slit $F$ is removed these coarse fringes disappear. The fringes visible through the slit have, however, both an inferior and superior limit in $e$ and in angular size. When $e$ is diminished to zero they vanish and when $e$ is sufficiently increased they agnin vanish, though they now appear when the slit is either removed or widened. From this it follows that the coarsest 
fringes come from the edges of the crack $S$ of the gratings, and that the remainder of the grating will not produce coarse fringes. By moving the slit the fringes may be made to appear in any other part of the spectrum.

The same fact may be proved by putting a vertical slit over the lens of the collimator and allowing white light to fall on the edges of the grating at $S$. Coarse fringes limited as to range and size are then seen throughout the spectrum.

Whenever the slit or vertical stop is used, the fringes are exceptionally sharp and easily controlled for micrometry. It is not even necessary to adjust the two spectra horizontally with the same care as when no slit is used; but the vertical coincidence of spectrum lines must be sharp. Naturally the use of the slit has one drawback, as the resolving power of the grating is decreased and the spectrum lines are only just visible. The adjustment, however, may be made before the slit is added. A fow examples may be given : For a slit $1 \mathrm{~mm}$. wide over the telescope or collimator, only the immediate edges at the crack $S$, about $1 / 2 \mathrm{~mm}$. each in breadth, are active. A narrow range of large fringes is seen in the field easily controlled by the micrometer screw. With a slit $3 \mathrm{~mm}$. in width the lower limit is much increased, the upper diminished, to a size of about $3^{\prime}$ of arc per fringe. In the absence of the slit the field is free from fringes. With a slit $6 \mathrm{~mm}$. wide, the upper limit is again decreased, the lower much increased; nevertheless the finest fringes appear only after the slit is removed. Using double slits over the collimator, each $1 \mathrm{~mm}$. wide and $3 \mathrm{~mm}$. apart, fringes of medium size limited at both ends appear; $3 \mathrm{~mm}$. slits $6 \mathrm{~mm}$. apart show only the very fine fringes, but both sizes are still limited. Finally, when all but about $1 / 2 \mathrm{~mm}$. of the edge of the crack of the grating $g^{\prime}$ is screened off, whereas the whole grating $g$ (about $1 / 2$ inch square) is without a screen, all the fringes from the maximum size to complete evanescence beyond the range of visibility are producible. Naturally if the edge of $g^{\prime}$ is quite dark everything vanishes.

It follows, therefore, that pairs of corresponding rays are always in question. These corresponding rays are at a definite distance, $\mathrm{ND}$, apart, where $\mathrm{D}$ is the grating space and $N$ the number of lines per centim. of the grating in question. This distance $\mathrm{ND}$ is greater as the fringes are smaller, and may be of the order of a centimetre when the fringes pass beyond the range of visibility. Again, ND is equal to the width of the crack when the largest fringes 
vanish. Finally, when ND is zero, as in the original unbroken grating, the size of the fringes is infinite.

It has been stated that the use of the slit or a laterally limited objective is advantageous because all the lines are much sharper. Inert or harmful illumination is cut off. If the slit is over the objective of the telescope only a small part of the field of view shows the lines; if placed over the objective of the collimator, the fringes are of extreme clearness throughout the spectrum. It may be ultimately of advantage to use the edge near the crack $g^{\prime}$ only, together with the whole of $g$. For if a small strip of $g^{\prime}$ at the crack $\mathrm{S}$ is used with the whole of $g$, the smaller fringes are weakened or wiped out. Thus the inner edge of the nearer grating with successive parts of the further grating is chiefly effective in the production of these interferences.

To bring the two edges quite together was not possible in my work, as they were rough and the apparatus improvised.

7. Data.--Some measurements were attempted, with the view only of checking the equations presently to be deduced. The adjustment on an ordinary spectrometer is not firm enough, and the fringes being very fine (a few minutes of arc) are difficult to follow unless quite stationary.

These observations gave both the values of $d e / d n$, displacement per fringe, for different angles of incidence $i$ and of diffraction $\theta$, and $d \theta / d n$, the angular deviation per fringe at the $\mathrm{D}$ line. In measuring the latter it was necessary to count the fringes between the $\mathrm{C}$ and $\mathrm{D}$ lines and divide their angular distance apart by these numbers. As $e$ cannot be measured, its successive increments $\Delta e$ from the first position must be used. These are presently to be associated with the corresponding increments of $d n / d \theta$. The data will be found in the original paper.

8. Equations.-In fig. 4 (p. 126), L and L' represent a pair of corresponding white rays, reflected into $R$ and $R^{\prime}$ and diffracted into $\mathrm{D}$ and $\mathrm{D}^{\prime}$ at angles $i$ and $\theta$, respectively. The half gratings $g$ and $g^{\prime}$ are separated along the crack $\mathrm{S}$, and $g^{\prime}$ is movable parallel to itself by a micrometer screw normal to $g^{\prime}$. Let the normal distance apart of the gratings be $e$. The incident rays $\mathrm{L}, \mathrm{L}^{\prime}$ strike the originally coplanar grating at points $\mathrm{N}$ rulings apart, or $\mathrm{ND} \mathrm{cm}$. apart, if $\mathrm{D}$ is the grating space. In the separated grating let these points be at a distance $c$ apart. Let $d$ be the incident wave front and $h$ the corresponding diffracted wave front, and call the angle between $c$ and $d, \gamma$. 
When there is reinforcement the path difference of the rays $L$ and $L^{\prime}$ from the incident $(d)$ to the diffracted $(h)$

Fig. 4.

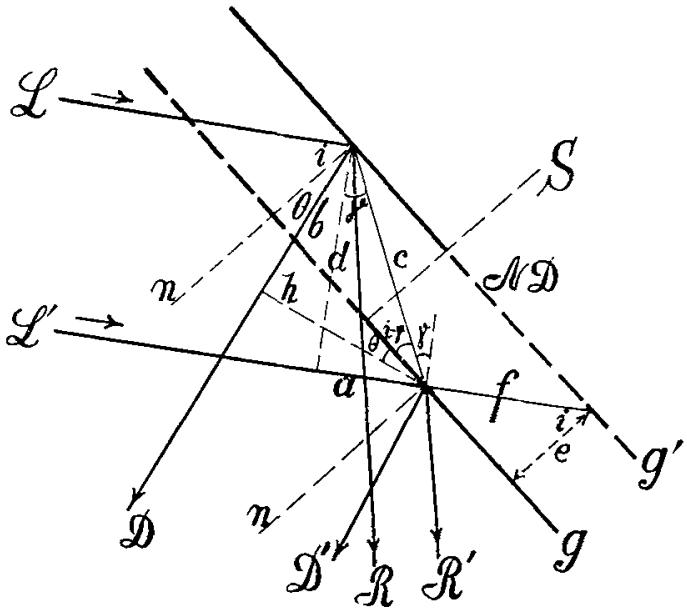

wave front, may be written

$$
n \lambda=b-a,
$$

where $b$ and $a$ are the distances of $h$ and $d$ from the points of incidence of $\mathrm{L}$ and $\mathrm{L}^{\prime}$ on the grating $g$ and $g^{\prime}$, respectively. If, finally, $f$ is the length of the prolongation of $\mathrm{L}^{\prime}$ between the gratings we may write in succession

$$
\begin{aligned}
& d=\mathrm{ND} \cos i, \quad \text {. . . . . } \\
& f=e \sec i, \text {. . . . . . . } \\
& a=\mathrm{ND} \sin i-e \sec i, \text {. . . . } \\
& \tan \gamma=a / d, \quad \text {. . . } \\
& c=\mathrm{ND} \cos i \sec \gamma, \text {. . . . . } \\
& b=c \sin (i+\theta-\gamma) \text {. . . . . }
\end{aligned}
$$

To these should be added

$$
d \mathrm{~N} / d e=\tan i / \text { D. . . . . . . . . }
$$

Hence, after removing $\gamma$, arranging and reducing,

$$
n \lambda=\mathrm{ND}(\sin \theta-\sin i)+e \sec i(1+\cos (i+\theta)) ;
$$

or since

finally,

$$
\sin i-\sin \theta=\lambda / \mathrm{D} \text {, }
$$

$$
(n+N) \lambda=e \frac{1+\cos (i+\theta)}{\cos i}=\frac{2 e \cos ^{2}(i+\theta) / 2}{\cos i} .
$$


This must, therefore, be regarded as the equation of the phenomenon. Equation (7) however leads on integration to

$$
\mathrm{N}=e \tan i / \mathrm{D}+\mathrm{N}_{0}, \quad . \quad . \quad . \quad . \quad \text {. }
$$

where $\mathrm{N}_{0} \mathrm{D}$ is the width of the crack.

If the value of $\mathrm{N}$ from (9) is put into (8) together with the equivalent of $\lambda / D$, it appears after reduction that

$$
\left(n+N_{0}\right) \lambda=e(\cos i+\cos \theta)=2 e \cos \frac{i+\theta}{2} \cos \frac{i-\theta}{2} \text {. }
$$

The case of $\mathrm{N}=0, e>0$ would correspond to the equation

$$
n \lambda=e(1+\cos (i+\theta)) / \cos i, \quad . \quad . \quad .
$$

which is only a part of the complete equation (8). In the case of $i>\theta$, one active half is necessarily partly behind the other half, and therefore not adapted to bring out the phenomenon as explained, unless $e=0$.

9. Differential Equations. Displacement per fringe, deldn. -To test equations (8) or (10) increments must be compared. The latter gives at once, since $\mathrm{N}$ is constant relative to $e$, like $i, \theta$, and $\lambda$,

$$
\frac{d e}{d n}=\frac{\lambda}{\cos i+\cos \theta}=\frac{\lambda}{2 \cos \frac{i+\theta}{2} \cos \frac{i-\theta}{2}}, .
$$

which is the interferometer equation when the fringes pass a given spectrum line, like either D line, which is sharp and stationary in the field. Equations (7) and (11), moreover, give after reduction

$$
d \mathrm{~N} / d n=\tan i \tan \frac{i-\theta}{2} . . . .
$$

Values of $d e / d n$ computed from (11) agreed as well with observations made under widely different conditions $(i>\theta$, $i<\theta$, first and second order), as the small fringes and the difficulty of getting the grating normal to the micrometer screw in my improvised apparatus, admit. If this adjustment is not perfect $\mathrm{N}_{0}$ changes with e. From equation (12) moreover,

$$
\frac{d \mathrm{~N}}{d n}=\frac{d \mathrm{~N}}{d \theta} \frac{d \theta}{d n}=\frac{d \mathrm{~N}_{0}}{d \theta} \frac{d \theta}{d n}=\frac{d \mathrm{~N}_{n}}{d n}, . . .
$$

since $\mathrm{N}_{0}$ is constant only relative to $e$ when $\theta$ varies.

10. Deviation per Fringe, \&c., $d \theta / d n, d \theta / d e$. - These measurements are still more difficult in the absence of special apparatus, since $e$ is not determinable and the counting of fine flickering fringes is unsatisfactory; but the order of 
results may be corroborated by observing the number of fringes between two Fraunhofer lines, like the C, D and other lines nsed. Differentiating equations (8) and (10) for variable $n, \lambda, \theta$, and $\mathrm{N}$ (since $d \mathrm{~N} / d \theta$ is equal to $d \mathrm{~N}_{0} / d \theta$, equation $12^{\prime}$ ), and inserting $-\mathrm{D} \cos \theta \cdot d \theta / d n=d \lambda / d n$, it follows, after arranging, that

$$
\frac{d \theta}{d n}=\frac{\lambda^{2}}{e \bar{D}} \frac{1+d \mathrm{~N} / d n}{1+\cos (i+\theta)}=\frac{\lambda}{e \cos i} \tan \frac{i-\theta}{2} .
$$

Combining this with (11),

$$
\frac{d \theta}{d n}=\frac{\lambda}{e \mathrm{D} \cos i}=\frac{\sin i-\sin \theta}{e \cos i} . . .
$$

Since in equation (13), $e$ is not determinable it is necessary to compare increments $\Delta d n / d \theta$ in terms of the corresponding increments $\Delta e$, whence

$$
\Delta(d n / d \theta)=\left(\cos i / \lambda \tan \frac{i-\theta}{2}\right) \Delta e . .
$$

My observations contain data of this kind, computed separately for the Fraunhofer lines D, C, \&c., employed and their mean values. To find the mean width of fringes between these lines, their angular deviations were divided by the number of fringes counted between them at different values of $e$. The results agree as closely as the difficulty of the observations varrants. One may note that without removing $\mathrm{N}$, the corresponding coefficients would be

$$
\Delta d(n+\mathrm{N}) / d \theta
$$

and these are found to be much more in error, here and in the preceding cases. If from $d \theta / d n, e$ is eliminated in terms of $(n+\mathrm{N})$ the equation is

$$
\frac{d \theta}{d n}=\frac{\lambda}{\mathrm{D}} \frac{1}{\left(n+\mathrm{N}_{0}\right) \cos i}, \quad . \quad . \quad . \quad .
$$

so that for a given value of $i, \theta, \mathrm{N}_{0}$, they decrease in size with $n$. If $n=0$ they reach the limiting size

$$
\frac{d \theta}{d n}=\frac{\lambda}{\mathrm{DN}_{0} \cos i} \text {. }
$$

If the crack $\mathrm{N}_{0} \mathrm{D}$ should be made infinitely small, they would be infinitely large. To pass through infinity, $\mathrm{N}_{0}$ must be negative, which has no meaning for $i>\theta$ or would place one effective edge of the crack $S$ behind the other. These inferences agree with the observations as above detailed. If, however, $i<\theta$, a negative value of $\mathrm{N}_{0}$ restores equation (16) 
for $n=0$ to equation (17), as was actually observed (figs. 2 and 3).

Finally, equation (14) might be used for observation in the incremented form

$$
\Delta(d e / d \theta)=\frac{\mathrm{D} \cos i}{\lambda} \Delta e ; . . .
$$

but I did not succeed with it. One loses track of the run of a fringe with $d e$.

11. Coloured Slit Images and Disk Colours of Coronas.In the above experiment the fringes were but a few minutes apart. It is obvious, however, that if $\lambda_{0}$ is sufficiently small the fringes will grow with decreasing $n$, in angular magnitude, until there are but a few black bands in the spectrum. Under these circumstances the undeviated image of the superimposed slits must appear coloured, particularly so, if an effect equivalent to $\mathrm{N}_{0}$ is present throughout the grating. This phenomenon of coloured slits is apparently of interest in its bearing on the theory of coronas, where there is also an interference phenomenon superimposed upon a diffraction phenomenon, as is evidenced by the brilliant disk colours. For instance, suppose a corona were produced by a sufficient number of fog particles distributed throughout a plane normal to the undeviated rays. Now let the alternate particles be moved in the same way slightly to the rear of their original position, and let the distance between the two planes be small relatively to the wave-length of light. In such a case there should be two identical coronas superimposed in all their parts, and they should therefore interfere. Inasmuch, however, as even small fog particles are of the order of size of $.0001 \mathrm{~cm}$. and their mean distance apart 50 times larger, i. e. $\cdot 005 \mathrm{~cm}$., it remains to be proved whether such an effect can be looked to as an explanation of the disk colours of coronas.

The phenomena of $\S 4$ must be producible with a transmission grating, separated parallel to the rulings, with one component grating movable micrometrically, though I bave not yet tested this case. Obviously it will only be interesting if obtainable from film gratings.

In this connexion I may state, in conclusion, that I have since succeeded in producing the elliptic interferences discussed in my last paper $(l . c$.$) , with such gratings, almost as brilliantly$ as with gratings ruled on glass. The film for this purpose is cemented, under pressure, with chloroformed Canada balsam, between unequal thicknesses of plate glass.

My thanks are due to Prof. A. A. Michelson, of the University of Chicago, for the loan of one of his reflexion gratings, with which the above results were tested.

Phil. Mag. S. 6. Vol. 22. No. 127. July 1911. 